\title{
Insights into Functional Mitral Regurgitation Using Transillumination Rendering
}

\author{
Roberto Ramirez ${ }^{1}$, Richard $\mathrm{Ro}^{2}$, Stamatios Lerakis ${ }^{3}$, and Edgar Argulian ${ }^{4}$ \\ ${ }^{1}$ Mount Sinai Morningside Hospital \\ ${ }^{2}$ Mount Sinai Roosevelt Hospital \\ ${ }^{3}$ Mount Sinai Medical Center \\ ${ }^{4}$ Mt Sinai Sch Med
}

December 15, 2020

\begin{abstract}
Case 1. An 82-year-old man with history of ischemic cardiomyopathy and multiple admissions due to acute decompensated heart failure was evaluated for moderate to severe secondary MR due to atrial dilation (atrial functional MR). TTE showed severe biatrial enlargement with a left atrial volume of $117 \mathrm{~mL}$ and a left atrial volume index of $65.5 \mathrm{ml} / \mathrm{m} 2$. It also showed LV of normal size, left ventricular LVIDd of $4.5 \mathrm{~cm}$ and LVEF of $55 \%$. En face view revealed two central jets arising from the coaptation gaps between posterior mitral leaflet indentations (P1/P2 and P2/P3) (Panel A). (Panel B) Transillumination rendering on 3D TEE, highlighted two distinct coaptation gaps between posterior mitral leaflet scallops. Case 2. A 63-years-old woman with medical history of ischemic cardiomyopathy and heart failure with reduced ejection fraction (35\%) was evaluated for moderate to severe secondary MR. TTE showed the LV dilation with LVIDd of $5.7 \mathrm{~cm}$. TEE revealed severe eccentric MR. (Panel C) 3D color Doppler TEE imaging of the mitral valve showed a severe regurgitant jet, originated in-between P2 and P3 posterior scallops. (Panel D) Transillumination rendering on 3D TEE, view from left atrium, in systole highlighted the coaptation gap. (Panel E) 3D color Doppler TEE imaging showed residual mild MR after a mitral clip was deployed grasping the medial aspect of P2 and A2 scallops covering the coaptation defect. (Panel F) Transillumination rendering on 3D TEE, view from LV, showed complete resolution of the coaptation gap between posterior scallops after clip deployment.
\end{abstract}

Title: Insights into Functional Mitral Regurgitation Using Transillumination Rendering

Brief title: Transillumination rendering in functional MR

Authors: Roberto H Ramirez, MD; Richard Ro, MD; Stamatios Lerakis, MD, PhD; Edgar Argulian, MD, $\mathrm{MPH}$.

Division of Cardiology, Mount Sinai Morningside, Mount Sinai Heart, Icahn School of Medicine at Mount Sinai, New York, New York

Address for correspondence:Dr. Roberto Ramirez, Mount Sinai Heart, Mount Sinai Morningside, Icahn School of Medicine at Mount Sinai, 1111 Amsterdam Avenue, New York, New York 10025.

Email: rhramirezmarmolejo@gmail.com

No conflicts of interest to disclose.

Case 1.

An 82-year-old man with history of ischemic cardiomyopathy and multiple admissions due to acute decompensated heart failure was evaluated for moderate to severe secondary MR due to atrial dilation (atrial 
functional MR). TTE showed severe biatrial enlargement with a left atrial volume of $117 \mathrm{~mL}$ and a left atrial volume index of $65.5 \mathrm{ml} / \mathrm{m} 2$. It also showed LV of normal size, left ventricular LVIDd of $4.5 \mathrm{~cm}$ and LVEF of $55 \%$. En face view revealed two central jets arising from the coaptation gaps between posterior mitral leaflet indentations (P1/P2 and P2/P3) (Figure A; Video A). (Figure B; Video B) Transillumination rendering on 3D TEE, highlighted two distinct coaptation gaps between posterior mitral leaflet scallops.

Case 2.

A 63-years-old woman with medical history of ischemic cardiomyopathy and heart failure with reduced ejection fraction (35\%) was evaluated for moderate to severe secondary MR. TTE showed the LV dilation with LVIDd of $5.7 \mathrm{~cm}$. TEE revealed severe eccentric MR. (Figure C; Video C) 3D color Doppler TEE imaging of the mitral valve showed a severe regurgitant jet, originated in-between P2 and P3 posterior scallops. (Figure D; Video D) Transillumination rendering on 3D TEE, view from left atrium, in systole highlighted the coaptation gap. (Figure E; Video E) 3D color Doppler TEE imaging showed residual mild MR after a mitral clip was deployed grasping the medial aspect of P2 and A2 scallops covering the coaptation defect. (Figure F; Video F) Transillumination rendering on 3D TEE, view from LV, showed complete resolution of the coaptation gap between posterior scallops after clip deployment.

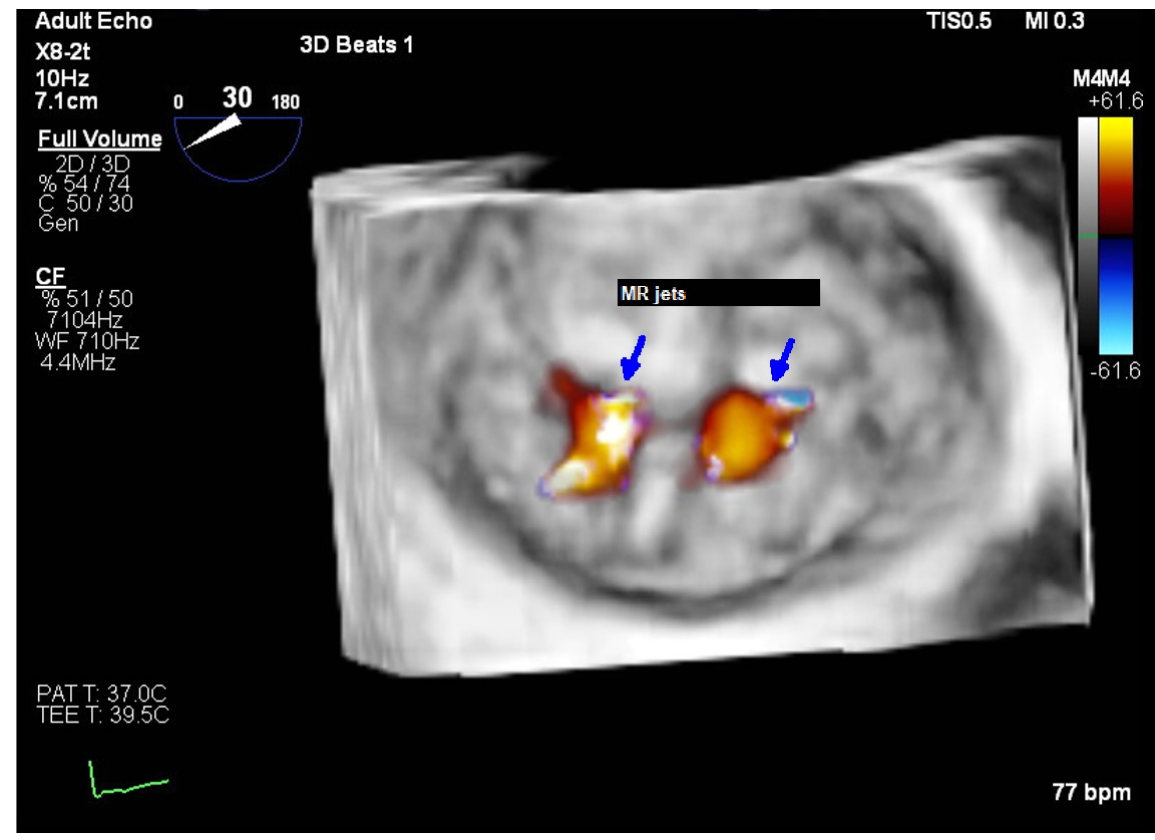



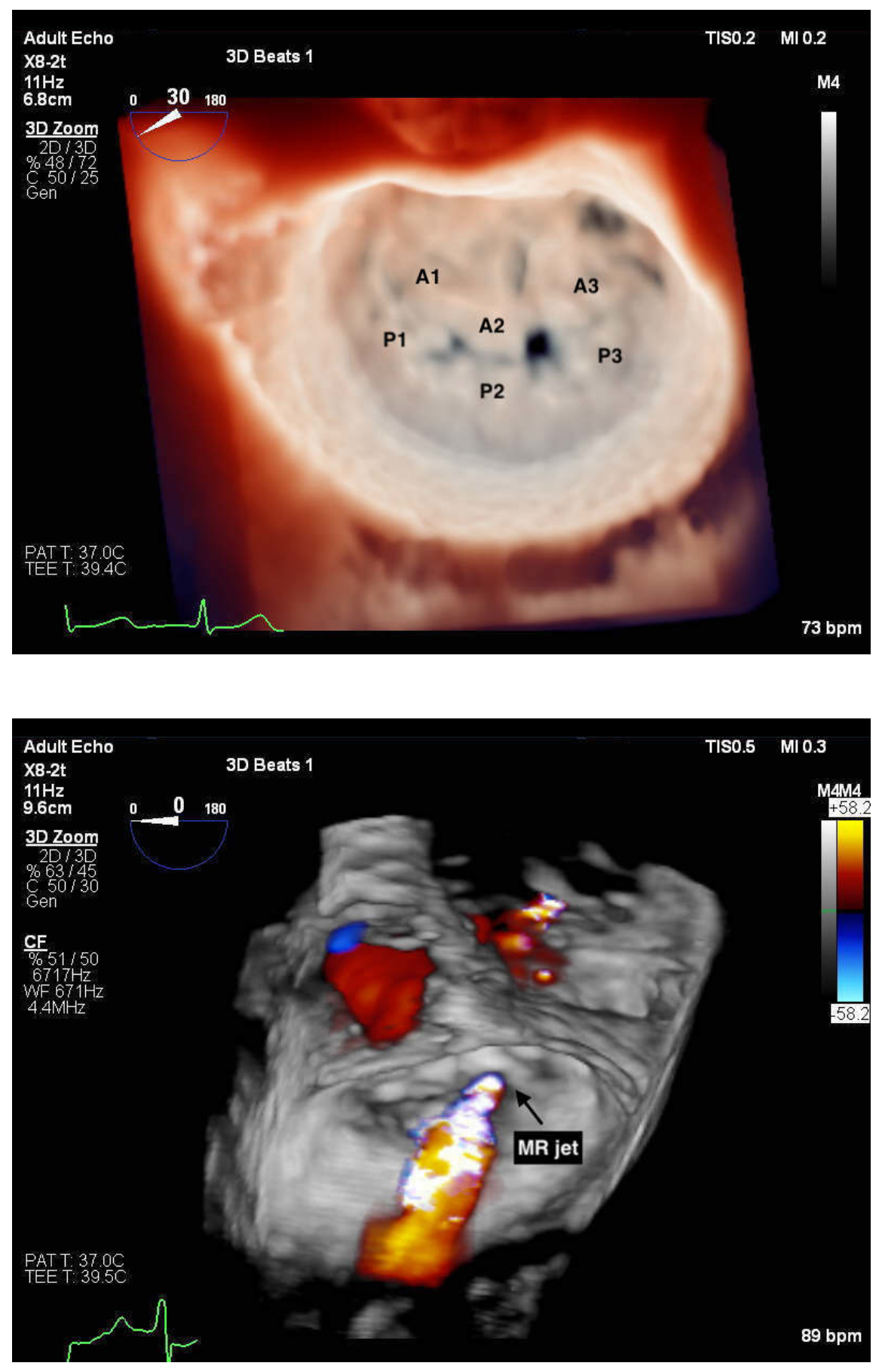

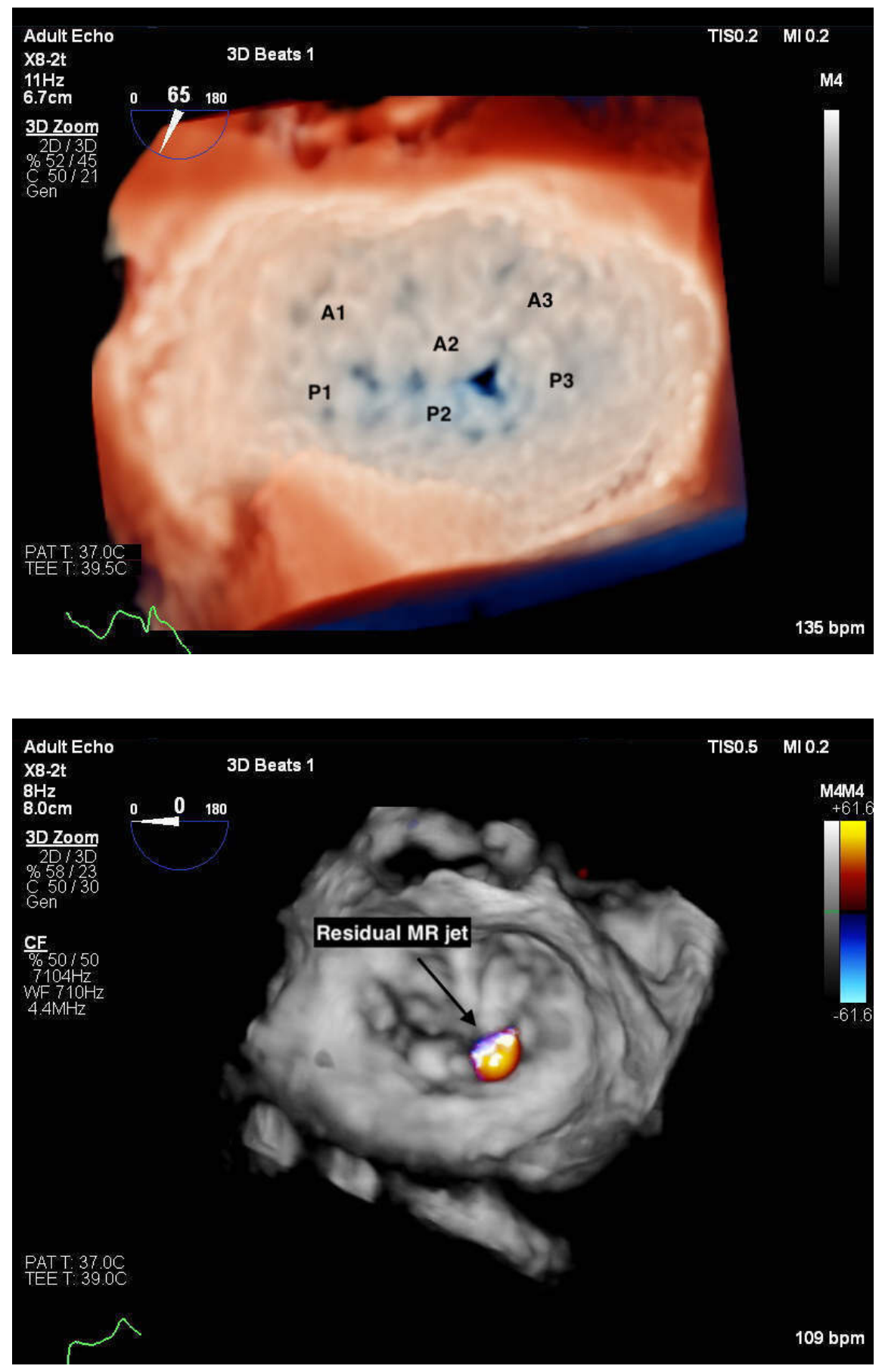


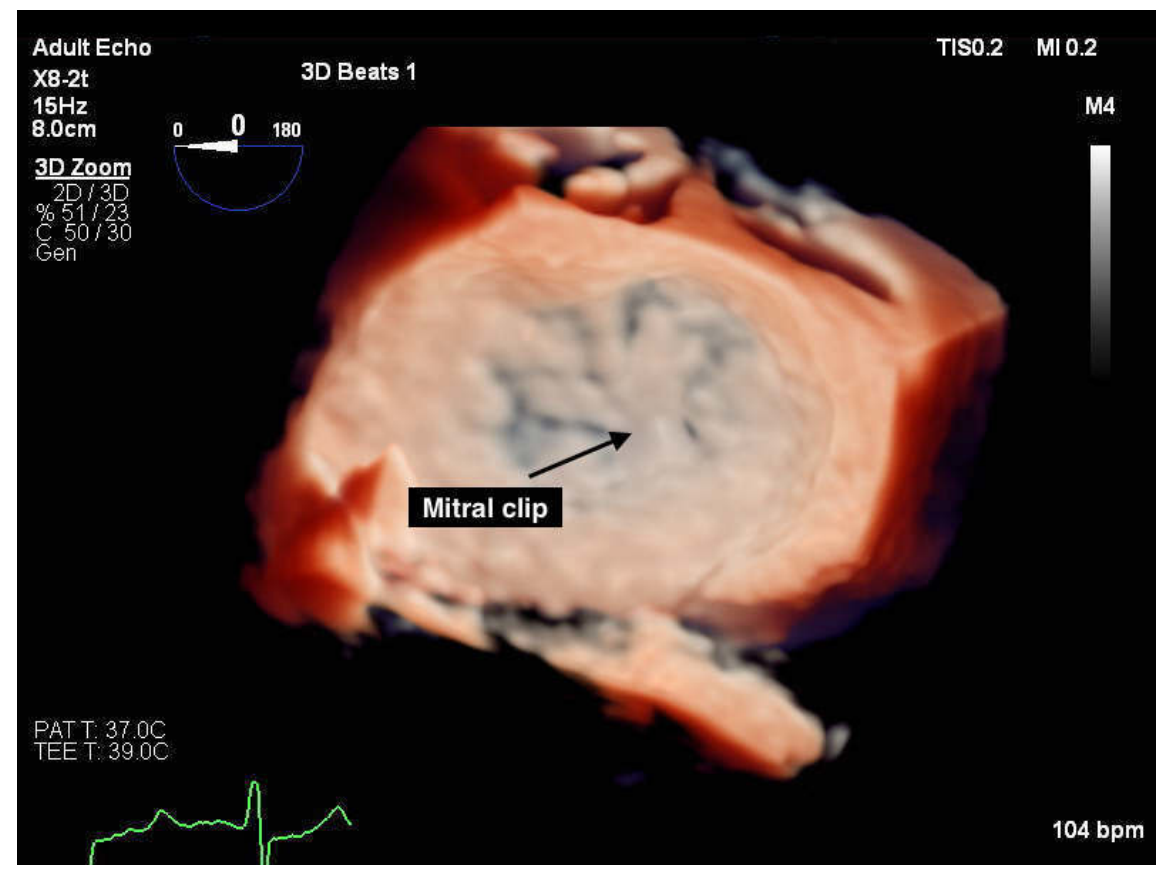

